\title{
Obstetrician injury whilst managing a shoulder dystocia: A case report
}

\author{
Sa’adatu Usman*, Srujana Parupalli, Amith Pinto, Nicholas Neal, Fidelma O’Mahony \\ University Hospital of North Staffordshire, Stoke-on-Trent, England \\ Email: sau691@yahoo.co.uk
}

Received 21 November 2011; revised 22 December 2011; accepted 20 January 2012

\begin{abstract}
Anecdotally, obstetricians have been known to sustain physical injuries whilst assisting deliveries but these are not always reported. This case describes how a mallet finger deformity was sustained by a consultant obstetrician whilst attempting to deliver a fetus with shoulder dystocia. Although this is a rare type of injury, it had significant impact on the consultant's ability to provide clinical care for some time. Disruptions in service provision have direct financial consequences to the healthcare system. This case report highlights the importance of reporting these injuries at the workplace.
\end{abstract}

Keywords: Shoulder Dystocia; Mallet Finger; Occupational Injuries

\section{CASE REPORT}

A 37 year old woman in her sixth pregnancy had shoulder dystocia at delivery. As per unit protocol, the emergency buzzer was activated and senior staff including the labour ward consultant responded promptly. When the shoulders could not be delivered by adopting the McRobert's position, the consultant took over the delivery. Rotation either clockwise or anti clockwise was unsuccessful. Whilst attempting to deliver the posterior arm with her right hand, an audible 'pop' was heard. The consultant withdrew her hand and on identifying a suspected mallet deformity handed the delivery over to the senior midwife. The delivery of the posterior arm was completed by the senior midwife with the aid of an episiotomy. A baby weighing $4320 \mathrm{~g}$ with APGARs of 2 at 1 minute and 8 at 5 minutes was subsequently delivered. Arterial cord gas $\mathrm{pH}$ was 7.13 with base excess of -4.9 .

An orthopaedic opinion confirmed the diagnosis of a mallet finger deformity with rupture of distal and middle inter phalangial extensor tendon. An x-ray was carried out to exclude bony injury and the clinician was treated

\footnotetext{
"Corresponding author.
}

with a rigid finger splint thus preventing her from operating for six weeks. An extensor lag persisted for a further 6 weeks. The extensor lag fortunately disappeared 4 months later.

\section{DISCUSSION}

Shoulder dystocia occurs 1 in 300 deliveries and is associated with a high perinatal mortality and morbidity even when managed appropriately [1]. The Confidential Enquiry into Stillbirths and Deaths in Infancy (CESDI) report in 1998 identified that $47 \%$ of the babies with shoulder dystocia died within 5 minutes of the head being delivered [2]. The McRobert's maneuver being the single most effective intervention should be performed first. If a McRobert's maneuver is unsuccessful internal manipulation is advised [3]. Delivery of the posterior arm is a recommended method of internal manipulation to manage shoulder dystocia [3-5]. In this case the patient exhibited several risk factors for shoulder dystocia, namely obesity and grand-mulitparity. Unfortunately these also increase the morbidity associated with caesarean section and so vaginal delivery was considered the safer option. It is therefore vitally important that staff on labour ward are competent in dealing with obstetric emergencies such as shoulder dystocia as per the clinical guidelines [3].

Apart from the biological, chemical, psychological and occupational hazards in obstetrics, there is growing evidence of physical injuries sustained at work. Musculoskeletal injuries comprise the second largest group [6]. Physical injuries such as a mallet finger deformity sustained by a clinician whilst performing manoeuvres for the delivery of the posterior arm has never been reported in the literature. This injury occurs due to rupture of the digital extensor tendon near its insertion into the distal phalynx or secondary to a fracture of the base of the distal phalynx [7]. Although commonly seen in athletes, it can also result from innocent trauma and can cause permanent disability if treatment is not sought or delayed [8].

Work place injuries have a major impact on staff sickness absences which in turn has a significant bearing on service provision within the healthcare system. The di- 
rect cost of this to the health service is over $£ 170 \mathrm{~m}$ a year [9]. Between April and June 2009, the average sickness absence rate for the NHS in England was 4.05\%. The highest numbers are reported in healthcare assistants, nursing and midwifery staff. Sickness absence is the least reported in medical and dental professionals. In the case described, the obstetrician was unable to carry out any clinical duties including on call and outpatient care for six weeks.

This case adds to the growing list of injuries sustained by obstetricians and other professionals in maternity care. Some common injuries include back strain, neck, hand and arm repetitive strain injuries sustained during operative deliveries. Currently there is no way of avoiding these injuries which can potentially lead to long term disability. Emergency Obstetric drills focus on maternal and fetal wellbeing, but perhaps training should be extended to encompass minimising risks to health care workers. Health care staff in general should take personal responsibility in reporting these injuries sustained at work so that they can be supported and advised by the occupational health department. Taking responsibility to report these incidents also implies probity and professionalism with respect to attitudes of health care workers. Reporting workplace injuries facilitates staff to obtain necessary support during their absence from work and when they return to work.

\section{REFERENCES}

[1] Gherman, R.B., Chauhan, S., Ouzounian, J.G., Lerner, H.,
Gonik, B. and Goodwin, T.M. (2006) Shoulder dystocia: The unpreventable obstetric emergency with empiric management guidelines. American Journal of Obstetrics and Gynaecology, 195, 657-672. doi:10.1016/j.ajog.2005.09.007

[2] Focus Group Shoulder Dystocia (1998) Confidential enquiries into stillbirths and deaths in infancy. Fifth annual report. Maternal and Child Health Research Consortium, London, 73-79.

[3] http://www.rcog.org.uk/resources/Public/pdf/shoulderdys tocia_42.pdf

[4] Naef, R.W. and Morrison, J.C. (1994) Guidelines for management of shoulder dystocia. Journal of Perinatology, 14, 435-441.

[5] Levy, B.S, Wegman, D.H., Baron, S.L. and Sohas, R.K. (2000) Occupational health, recognising and preventing work related diseases and injury. 4th Edition, Williams and Wilkins, Lippincot.

[6] Mallet fracture. http://emedicine.medscape.com/article/98168-overview

[7] Maitra, A. and Dorani, B. (1993) The conservative treatment of mallet finger with a simple splint: A case report. Archives of Emergency Medicine, 10, 244-248.

[8] National Audit Office (2003) A safer place to work: Improving the management of health and safety risks to staff in NHS trusts. National Audit Office Report.

[9] Sickness Absence Rates in the NHS (2009) Experimental statistics. The NHS Information Centre, Workforce and Facilities Team. 